\title{
Networking
}

\section{Joint working to develop R\&D capacity in three rural primary care trusts}

Steve Robertson Institute for Health Research, HRDNoW Lancaster University, UK, Claire Hornby and Rod Jones Cumbria Primary Care Research Network, CambReN, UK

\begin{abstract}
Whilst much good practice relating to research support for rural PCTs undoubtedly exists, little has been published about the processes that facilitate such successful support. This paper outlines the nature of a collaborative venture between a Research \& Development Support Unit, HRDNoW, and a Primary Care Research Network, CumbReN in providing research support to three rural PCTs in North Cumbria. In doing so, the paper highlights how research capacity is built through a combination of inputs at an individual and organisational level and looks specifically at the outcomes of this collaboration.
\end{abstract}

Key words: collaboration; research capacity; research support; rural

\section{Introduction}

Historically, there has been a low research base and culture within many primary care organizations (NHS Executive, 1997). This stands in contrast to a recognition that good quality research, and its application, in primary care is much needed (De Maeseneer et al., 2003) and often neglected as a responsibility of PCTs (Department of Health, 2004). The starting point in solving this apparent contradiction must therefore lie in developing quality research capacity so that, as a minimum, NHS staff understand the research process and principles to enable the implementation of evidence-based practice. This paper considers how collaboration between a small primary care research network (Cumbria Primary Care Research Network, CumbReN) and a research and development support unit (Health R\&D North West, HRDNoW) has begun this process of research capacity development across three rural primary care trusts (PCTs).

Address for correspondence: Steve Robertson, Institute for Health Research, Lancaster University, Lancaster, LA1 4YT, UK. Email: s.robertson@lancaster.ac.uk

\section{Historical development}

As separate organizations both CumbReN and HRDNoW have been in existence for a number of years. CumbReN was first formed in 1988. Its aim has always been to provide an efficient and effective support network for all interested healthcare researchers in what are now the three North Cumbria PCTs: Carlisle and District PCT, Eden Valley PCT and West Cumbria PCT (Figure 1). HRDNoW, established in 1998, is a collaboration of three universities: Lancaster University (which hosts the core unit), the University of Liverpool and the University of Salford (Figure 2). It was established to provide research support to the NHS in what was the North West Regional Health Authority. At this time, research support for North Cumbria was the responsibility of the Northern and Yorkshire Regional Health Authority. With the disestablishment of the regions in 2001, HRDNoW's remit was extended and, for the first time, incorporated the North Cumbria PCTs. These changes provided the opportunity for $\mathrm{CumbReN}$ and HRDNoW to work collaboratively in developing research capacity within North Cumbria 


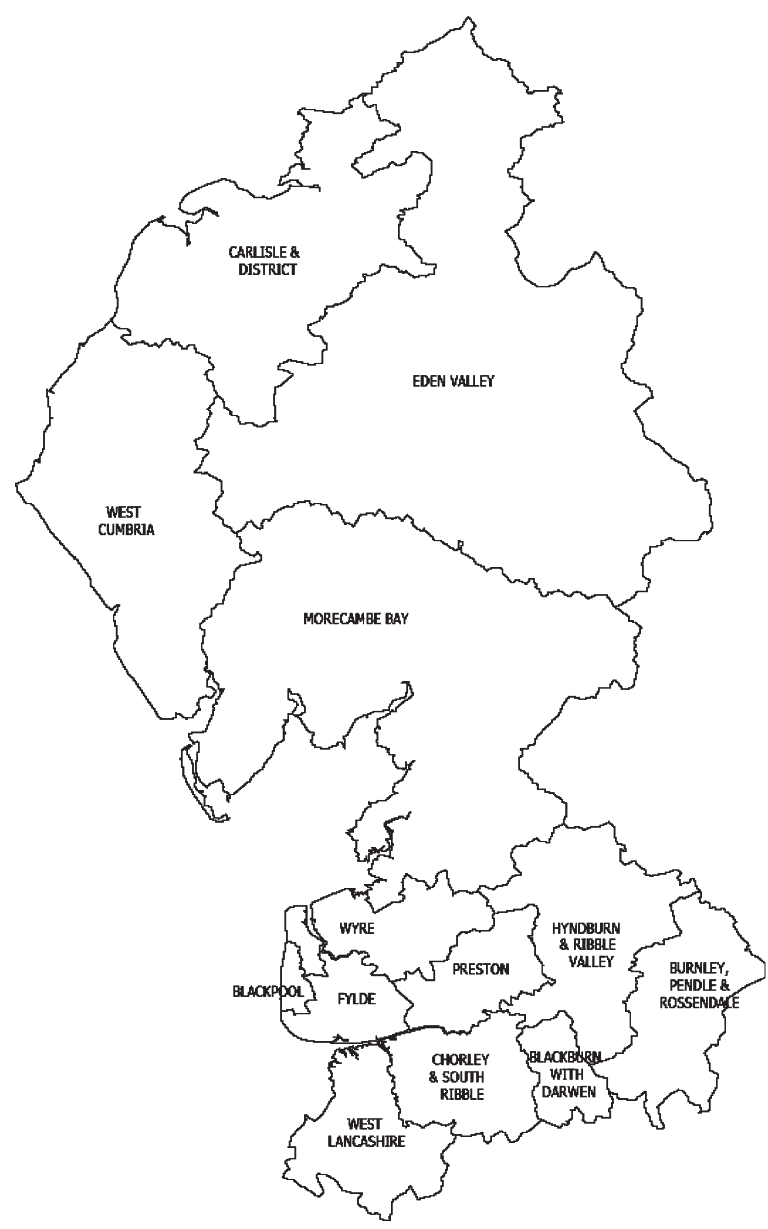

Figure 1 PCTs in Cumbria and Lancashire Strategic Health Authority

\section{The emerging relationship}

North Cumbria has a characteristically rural nature - a large geographical area (Figure 1) with low population density and attendant health issues (Cox, 1997). This creates both barriers and opportunities when considering $R \& D$ capacity and capability. The importance of collaborations between practitioners and academic institutions in developing an NHS research culture has been highlighted by Pitkethly and Sullivan (2003) and by Thomas et al. (2001). Yet, obtaining research support from academic institutions can be financially costly. Add to this the fact that no university has its main base in Cumbria (although three universities do have campus sites there) and issues of accessing such support and developing such necessary collaborations are compounded within this rural setting. Previous work undertaken by CumbReN had highlighted the need for high quality research education and training and, importantly, that this needed to be delivered locally. However, CumbReN only receives infrastructure support and does not have any dedicated funding to deliver such training. Early discussions between CumbReN and HRDNoW therefore focused on how and what research training could be developed and delivered locally to health professional staff given that such provision falls within the brief of HRDNoW. A rolling programme of bespoke workshops has developed since 2002 (Table 1) being convened by HRDNoW who act as a conduit, drawing in academic staff from appropriate disciplines to deliver these sessions. The programme now develops responsively in line with workforce-identified $\mathrm{R} \& \mathrm{D}$ training needs

\section{Moving forward}

Whilst the development of the workshop programme has been significant in itself, it has also cemented the collaboration between CumbReN and HRDNoW and has thereby led to a range of other positive outcomes. First, it has raised the profile of CumbReN as the first-line provider of research support for primary health care professionals. Second, potential researchers now have more ready and free access to what has become a known provider of R\&D expertise across a wide range of research topics, including statistical support from medical statisticians. Third, if this expertise is not directly available within HRDNoW, they will broker relationships with appropriate academics/departments on behalf of NHS staff.

Department of Health information on implementing Research Management and Governance (RM\&G) in primary care (Department of Health, 2002), states that existing expertise and resources, including research networks, should be utilized to develop research governance arrangements. Also, the same Department of Health information suggests that Research and Development Support Units (RDSUs) can have a significant role in RM\&G support. In North Cumbria, CumbReN 


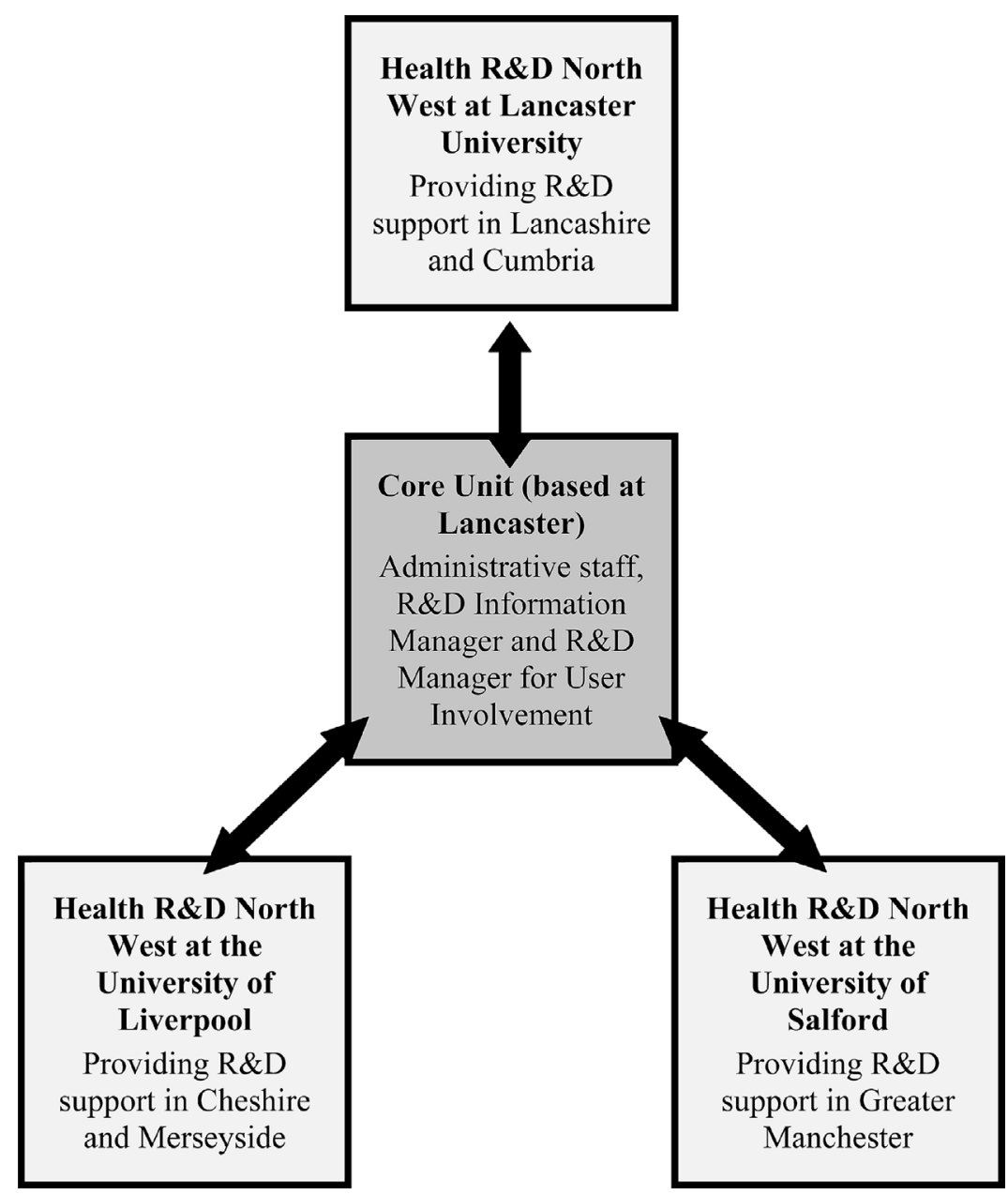

Figure 2 Structure of HRDNoW

provides RM\&G support to the PCTs but, through its close relationship with HRDNoW, it does not need to do so in isolation. HRDNoW facilitates peer support meetings for R\&D managers across the Lancashire and Cumbria Strategic Health Authority who are involved in implementing $\mathrm{RM} \& \mathrm{G}$ within their organizations. These meetings have enabled the support that CumbReN provides to the PCTs to be informed by best practice from other often larger and more research experienced organizations. There is also currently an emerging desire within Lancashire and Cumbria to develop shared RM\&G documentation and arrangements, for example with cross-organizational audit and monitoring of research. It is hoped that this will streamline what is often perceived as a complex and bureaucratic research process and thereby encourage more research within the two neighbouring counties.

In addition, the close relationship, established initially through the workshop programme, has also led to the involvement of HRDNoW at a strategic level within both CumbReN and the PCTs. A member of HRDNoW sits on the CumbReN education subcommittee and advises not only on future workshop developments but also provides practical input into the annual North Cumbria Health Care R\&D conference 
Table 1 Bespoke workshop programmes

2002

- Ethics and ethical approval

- Research governance

2003

- Ethics and ethical approval

- Introduction to surveys

- Accessing and critically reviewing research evidence

2004

- Involving service users in research and development

- Introduction to qualitative research

- Action research

- Understanding and undertaking focus groups and interviews

- Developing a research proposal

- Accessing and critically reviewing research evidence

organized by CumbReN. This input includes suggesting and approaching appropriate keynote speakers, reviewing abstract submissions, and chairing sessions on the day. A recent development within the PCTs is that they now have a shared corporate management team, including having a single chief executive. Under this new structure, an R\&D committee has been established that sits across the three PCTs. HRDNoW have been invited to sit on this committee, and their expertise can therefore be utilized to provide organizational development advice to help guide the strategic direction of R\&D within North Cumbria.

\section{Conclusion}

Since beginning collaborative working in 2002, a synergy has developed between CumbReN and HRDNoW that has helped build research capacity at an individual and organizational level within the North Cumbria PCTs. The following are specific outcomes of this process:
1) An increase in the provision of high quality research workshops tailored to workforce requirements.

2) A raised profile for both CumbReN and HRDNoW as providers of research support.

3) Easier access to appropriate academic expertise.

4) Assistance for the PCTs to receive greater support with implementing RM\&G arrangements, including the streamlining and standardization of these processes.

5) The opportunity for the PCTs to utilize organizational development expertise to help guide R\&D strategic direction across North Cumbria when needed.

Whilst much has been achieved, there is further work to be done and the changing nature of the NHS will undoubtedly bring new challenges. However, a relationship has been established between a small research network and an RDSU that will strengthen the ability of the organizations jointly served in North Cumbria to meet such challenges.

\section{References}

Cox J. 1997: Rural general practice: a personal view of current key issues. Health Bulletin 55, 309-15.

De Maeseneer, J.M., van Driel, M.L., Green, L.A. and van Weel, C. 2003: The need for research in primary care. The Lancet 362, 1314-19.

Department of Health. 2002: Development of research management and governance in primary and community care: information for primary care trusts. London: Department of Health.

Department of Health. 2004: Joint ministerial review of the role of primary care trusts in relation to learning and research in the new NHS. London: Department of Health.

NHS Executive. 1997: $R \& D$ in primary care. National Working Group Report. Professor David Mant, London: Department of Health.

Pitkethly, M. and Sullivan, F. 2003: Four years of TayRen, a primary care research and development network. Primary Health Care Research and Development 4, 279-83.

Thomas, P., Griffiths, F., Kai, J. and O'Dwyer, A. 2001: Networks for research in primary care. British Medical Journal $322,588-90$. 